\title{
Diffusion Tensor Imaging of Idiopathic Normal Pressure Hydrocephalus: A Voxel-Based Fractional Anisotropy Study
}

\author{
Tetsuo Koyama, ${ }^{1}$ Kohei MARUmoto, ${ }^{2}$ Kazuhisa DOMEn, ${ }^{2}$ \\ Takehisa OHMURA, ${ }^{3}$ and Hiroji MIYAKE ${ }^{3}$ \\ Departments of ${ }^{1}$ Rehabilitation Medicine and ${ }^{3}$ Neurosurgery, \\ Nishinomiya Kyoritsu Neurosurgical Hospital, Nishinomiya, Hyogo; \\ ${ }^{2}$ Department of Physical Medicine and Rehabilitation, \\ Hyogo College of Medicine, Nishinomiya, Hyogo
}

\begin{abstract}
Diffusion tensor imaging (DTI) using a 3.0 tesla magnetic resonance scanner was used to investigate white matter changes caused by idiopathic normal pressure hydrocephalus (INPH) in 10 patients diagnosed by clinical symptoms (gait disturbance, dementia, and/or urinary incontinence) and Evans index $>0.3$, and compared with findings for 10 age-matched controls ( $\geq 60$ years). Then, using a computer-automated method, fractional anisotropy (FA) brain maps were generated and finally transformed into the standard space. Voxel-based FA values within two regions of interests (ROIs), the forceps minor and corticospinal tracts, were then separately evaluated. Within each ROI, statistical comparisons of results from the INPH and control groups were performed. In addition, for INPH patients, grading scores for clinical symptoms and FA values were correlated. The forceps minor mean FA value was much smaller for the INPH group (0.504) than for the control group (0.631). The corticospinal tract mean FA value was slightly smaller for the INPH group (0.588) than for the control group (0.632). Additional analyses indicated that lower FA values within the forceps minor tended to be associated with clinical symptoms such as urinary incontinence and gait disturbance. Our findings indicate F A values decreased in the forceps minor of INPH patients. We also found that lower values were associated with severer clinical symptoms, implying that DTI techniques may be developed for more accurate diagnosis.
\end{abstract}

Key words: dilatation, elderly, neuroimaging, shunt, ventricle

\section{Introduction}

Idiopathic normal pressure hydrocephalus (INPH) is a neural syndrome that afflicts elderly people. The clinical manifestations are gait disturbance, dementia, and urinary incontinence without preceding disorders. ${ }^{1)}$ Patients with these symptoms often need care to cope with the activities of daily living, ${ }^{8,21)}$ so adequate diagnosis and treatment (e.g., ventriculoperitoneal shunt or lumbo-peritoneal shunt ${ }^{28)}$ are essential to reduce medical and social-welfare costs in countries with aging populations. The diagnosis is based on clinical observations supported by computed tomography (CT) and magnetic resonance (MR) imaging, and other neuroradiographical examina-

Received August 10, 2011; Accepted September 21, 2011 tions. ${ }^{4)}$

Ventricular dilation is a critically important neuroradiographical finding: an Evans index, the ratio of the maximum width of the frontal horns to the maximum width of the inner table of the skull, of greater than 0.3 is accepted as an essential diagnostic criterion for INPH. ${ }^{31,32)}$ In addition, recent MR imaging studies have indicated that tightness of the high parietal convexity is commonly observed in INPH patients. ${ }^{5,18,35)}$ Such anatomical changes in the cerebrospinal fluid (CSF) space have been widely accepted as neuroradiographical evidence justifying the diagnosis of INPH.

Detection of deep white-matter lesions is another important neuroradiographical finding commonly observed in INPH patients. ${ }^{24,32)}$ Diffusion tensor imaging (DTI), a newly developed MR technique, has been recently applied to evaluate white matter de- 
generation in INPH patients. ${ }^{6,9,20,22,27)}$ DTI can sensitively detect the direction of diffusion of water molecules and so reveals the orientation of the white matter tracts. ${ }^{36)}$ This anatomical detail enables clinically useful characterization of the degeneration of neural fibers affected by various neural diseases. ${ }^{37)}$ Recent studies based on fractional anisotropy (FA) values in some brain regions, including the corpus callosum and corticospinal tracts, have reported that patients with INPH exhibit white matter changes. ${ }^{6,20,22,27)}$ Quantitative analysis of FA values would be useful to better characterize the white matter changes in INPH patients. ${ }^{6,20)}$

The present study used a computer-automated technique for FA evaluation and showed that, compared with control subjects in a similar age range, FA values in the forceps minor (anterior portion of corpus callosum) were much lower in INPH patients and that low values were associated with more severe clinical manifestations.

\section{Methods}

This study recruited both INPH patients and control subjects from patients who visited our outpatient clinic between May 2010 and May 2011. All subjects (or, if more appropriate, relatives) provided written consent for inclusion in the study.

The INPH patients presented with gait disturbance or dementia or urinary incontinence or combinations of these symptoms. Our routine protocol included MR imaging, electrocardiography, chest radiography, and blood sampling. After obtaining informed consent, eligible patients then admitted to our hospital and underwent removal of $30 \mathrm{ml}$ CSF via a lumbar puncture (tap test). ${ }^{5}$ The inclusion criteria were age $\geq 60$ years, presence of one or more INPH symptoms, Evans index greater than 0.3, absence of known disorders causing ventriculomegaly, and normal CSF findings (protein $\leq 50 \mathrm{mg}$, cell count $\leq 3 \mathrm{~mm}^{3}$, and pressure $\left.\leq 20 \mathrm{cmH}_{2} \mathrm{O}\right)^{5}$; ; such criteria were identical to possible INPH as stipulated in the Japanese guidelines for INPH. ${ }^{4}$

The control subjects had chief complaints of headache or dizziness or both, but with no meaningful findings after medical examinations such as CT and blood analysis, and whose symptoms were alleviated within a 2-week follow-up period. Other inclusion criteria were age $\geq 60$ years, absence of neurological symptoms, and no history of neurological disorder.

MR imaging was typically performed 2 to 4 weeks after the initial visit to our outpatient clinic, using a 3.0 tesla MR scanner (Trio; Siemens AG, Erlangen, Germany) with a 32-channel head coil. The DTI scheme included acquiring 12 images with non-collinear diffusion gradients $\left(b=1000 \mathrm{sec} / \mathrm{mm}^{2}\right)$ and one non-diffusion-weighted image $\left(b=0 \mathrm{sec} / \mathrm{mm}^{2}\right)$, employing a single-shot echo-planar imaging sequence. A total of 64 axial slices were obtained from each patient. The field of view was $230.4 \mathrm{~mm} \times$ $230.4 \mathrm{~mm}$, the acquisition matrix was $128 \times 128$, and the slice thickness was $3 \mathrm{~mm}$ without a gap, which resulted in voxel-dimensions of $1.8 \mathrm{~mm} \times 1.8$ $\mathrm{mm} \times 3.0 \mathrm{~mm}$. Echo time was $83 \mathrm{msec}$ and repetition time was $7000 \mathrm{msec} . \mathrm{T}_{2}$-weighted images were obtained using a spin-echo sequence. The field of view was $218.88 \mathrm{~mm} \times 218.88 \mathrm{~mm}$, the acquisition matrix was $192 \times 192$, and the slice thickness was 3 $\mathrm{mm}$ without a gap, which resulted in voxel-dimensions of $1.14 \mathrm{~mm} \times 1.14 \mathrm{~mm} \times 3.0 \mathrm{~mm}$. Echo time was $88 \mathrm{msec}$ and repetition time was $5000 \mathrm{msec}$. A total of 50 axial slices were obtained from each patient. In addition, $\mathrm{T}_{1}$-weighted $\mathrm{MR}$ images were also obtained for other diagnostic purposes. The total time for MR imaging acquisition was approximately 20 minutes per patient. Patients whose inability to stay still prevented complete MR imaging acquisition were also excluded from our database.

The brain image analysis package FMRIB Software Library (FSL), comprising various tools including Brain Extraction Tool (BET), FMRIB's Diffusion Toolbox (FDT), FMRIB's Linear Image Registration Tool (FLIRT), FMRIB's Nonlinear Image Registration Tool (FNIRT), FSLUTILS (miscellaneous FSL utilities for converting and processing images), and FSLView (interactive display tool for three-dimensional and four-dimensional data), was used for image processing. ${ }^{16,34)}$ The FDT tool was used to align all images in volumetric relation to the first image ( $\left.b=0 \mathrm{sec} / \mathrm{mm}^{2}\right)$, and DTI data were corrected for motion and eddy current distortion. ${ }^{17)}$ Then, extra-cerebral matter was excluded from the images using the BET tool. ${ }^{15)}$ Next, to evaluate tensor diffusion and calculate each patient's brain FA values, DTI was analyzed using the FDT tool (FA brain map). Using FLIRT and FNIRT, ${ }^{13,14)}$ the FA values were mapped to standard stereotaxic space (International Consortium of Brain Mapping [ICBM] DTI-81 Atlas). ${ }^{19,29)}$ To keep the transformation procedure simple, as recommended in the manual, this study employed standard tasks settings for FNIRT. ${ }^{13)}$ Spatial transformations of the FA brain maps were confirmed by visual comparison with images generated by FSLView. ${ }^{12)}$ The same image processing procedures were used as in our previous study. ${ }^{23)}$

This study employed the ICBM DTI-81 Atlas to establish the standard stereotaxic space. ${ }^{19,29)}$ Using FSLUTILS, ${ }^{11)}$ the forceps minor (anterior portion of 

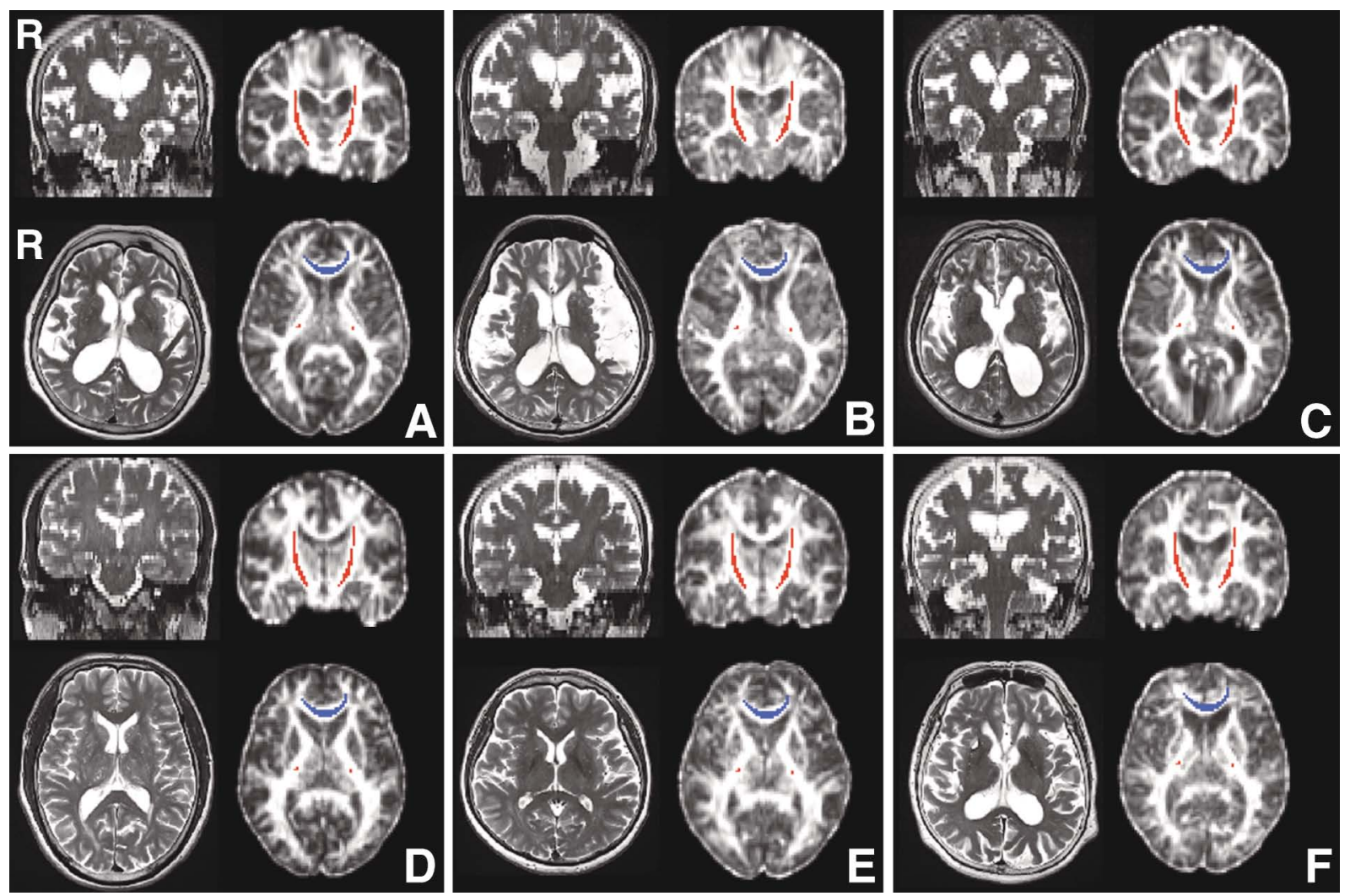

Fig. 1 Representative magnetic resonance images for typical cases. $T_{2}$-weighted images (left column) and fractional anisotropy (F A) brain images in standard space (right column). Region of interest is shown in blue (forceps minor) or red (corticospinal tracts). A: Case 1. B: Case 5, C: Case 10, D: Case 11, E: Case 15, F: Case 20.

corpus callosum) white matter tracts and bilateral corticospinal tracts were separately abstracted from the ICBM DTI 81 brain map (Fig. 1). FA values within regions of interest (ROIs) were assessed and mean values for single voxels were then calculated using FSLUTILS.

To classify the symptoms of INPH patients, and to score cognitive, gait, and urinary functions, we employed the INPH grading scale (from 0 , normal to 4 , severe disability), ${ }^{25)}$ and the modified Rankin scale (mRS) to score activities of daily living (from 0, normal to 5 , severe disability). ${ }^{33)}$

Effects of the tap test were evaluated from beforeand-after results of the 3-meter up-and-go tests. ${ }^{30)}$ These tests were conducted on the day before, and 3 hours and 1 week after the tap test. To index the effects of the tap test, we evaluated improvements in the up-and-go test results by calculating the ratio between best performance (in seconds) either 3 hours or 1 week after the tap test and the performance value before. Besides the up-and-go test, we also performed mini-mental state examination (MMSE) ${ }^{3)}$ and scored the frontal assessment battery (FAB). ${ }^{2)}$ For clarity, results from MMSE and FAB were not included in the analytical database.

For comparisons between the INPH group and the control group, conventional t-tests were separately performed within ROIs (forceps minor and corticospinal tracts). To evaluate the relationships between clinical symptoms and FA values in INPH patients, Spearman's rank correlation tests were used to compare FA values and INPH grading scale and mRS scores separately within ROIs. P values of less than 0.05 were considered statistically significant.

\section{Results}

Table 1 presents the patient profiles. Age ranged from 69 to 82 years (mean \pm standard deviation 75.1 \pm 4.5 years) for INPH patients and from 61 to 79 years (68.8 \pm 7.5 years) in the control group. Table 2 shows the tap test results. Except for Case 3, all patients showed more than $10 \%$ improvement (ratio $<0.9$ ) in up-and-go time after the tap test.

Figure 1 shows MR imaging findings for typical cases. $\mathrm{T}_{2}$-weighted imaging clearly showed ventricular dilation in patients with INPH symptoms. FA 
Table 1 Patient profiles

\begin{tabular}{|c|c|c|c|c|c|c|c|}
\hline $\begin{array}{l}\text { Case } \\
\text { No. }\end{array}$ & $\begin{array}{l}\text { Age } \\
\text { (yrs) }\end{array}$ & Sex & Group & $\mathrm{mRS}$ & $\begin{array}{c}\text { INPH } \\
\text { grading scale* }\end{array}$ & \multicolumn{2}{|c|}{ FA value } \\
\hline 1 & 79 & $\mathrm{~F}$ & INPH & 3 & 2: $2: 1$ & 0.582 & 0.642 \\
\hline 2 & 74 & $\mathrm{~F}$ & INPH & 2 & 1: $1: 1$ & 0.561 & 0.565 \\
\hline 3 & 70 & M & INPH & 2 & 0: $2: 1$ & 0.557 & 0.632 \\
\hline 4 & 77 & M & INPH & 4 & 3: $3: 3$ & 0.543 & 0.598 \\
\hline 7 & 72 & M & INPH & 3 & 1: $2: 3$ & 0.477 & 0.602 \\
\hline 8 & 81 & $\mathrm{~F}$ & INPH & 4 & 1: $3: 3$ & 0.476 & 0.577 \\
\hline 9 & 69 & M & INPH & 4 & 1: $3: 3$ & 0.471 & 0.558 \\
\hline 10 & 73 & $\mathrm{~F}$ & INPH & 4 & 3: $3: 3$ & 0.444 & 0.562 \\
\hline 11 & 67 & M & control & - & - & 0.685 & 0.671 \\
\hline 16 & 79 & $\mathrm{M}$ & control & - & - & 0.626 & 0.614 \\
\hline 17 & 79 & $\mathrm{~F}$ & control & - & - & 0.604 & 0.632 \\
\hline 18 & 64 & $\mathrm{~F}$ & control & - & - & 0.600 & 0.622 \\
\hline 19 & 70 & $\mathrm{M}$ & control & - & - & 0.556 & 0.651 \\
\hline 20 & 79 & $\mathrm{~F}$ & control & - & - & 0.525 & 0.625 \\
\hline
\end{tabular}

Separately within each group, patients are ordered according to forceps minor fractional anisotropy (FA) value (greater to smaller). Modified Rankin scale (mRS) and idiopathic normal pressure hydrocephalus (INPH) grading scale were assessed on the first visit to our outpatient clinic. ${ }^{*}$ Scores for cognitive impairment: gait disturbance: urinary incontinence. F: female, M: male.

Table 2 Tap test results

\begin{tabular}{rcccc}
\hline $\begin{array}{c}\text { Case } \\
\text { No. }\end{array}$ & $\begin{array}{c}\text { Before } \\
\text { (sec/steps) }\end{array}$ & $\begin{array}{c}\text { 3 Hours after } \\
\text { (sec/steps) }\end{array}$ & $\begin{array}{c}\text { 1 Week after } \\
\text { (sec/steps) }\end{array}$ & Ratio \\
\hline 1 & $21.3 / 35$ & $\mathbf{1 5 . 3} / \mathbf{3 0}$ & $21.4 / 36$ & 0.71 \\
2 & $13.2 / 23$ & $12.7 / 22$ & $\mathbf{1 1 . 6 / 2 0}$ & 0.88 \\
3 & $10.0 / 15$ & $\mathbf{9 . 5} / \mathbf{1 4}$ & $9.8 / 14$ & 0.95 \\
4 & $22.5 / 42$ & $\mathbf{1 9 . 8 / 3 9}$ & $24.5 / 47$ & 0.88 \\
5 & $33.1 / 50$ & $35.6 / 67$ & $\mathbf{2 3 . 3 / 4 3}$ & 0.70 \\
6 & $17.0 / 21$ & $\mathbf{1 3 . 3 / 2 0}$ & $16.4 / 22$ & 0.78 \\
7 & $11.5 / 24$ & $\mathbf{9 . 6} / \mathbf{1 9}$ & $9.9 / 23$ & 0.83 \\
8 & $37.1 / 49$ & $30.7 / 39$ & $\mathbf{2 8 . 6 / 4 3}$ & 0.77 \\
9 & $18.3 / 26$ & $14.9 / 23$ & $\mathbf{1 4 . 1 / 2 3}$ & 0.77 \\
10 & $43.9 / 72$ & $47.7 / 70$ & $\mathbf{3 6 . 8} / \mathbf{6 3}$ & 0.84 \\
\hline
\end{tabular}

Best performance after the tap test shown in bold.

brain images from INPH patients were successfully transformed into the standard space despite the potentially confounding effects of altered morphology, comparable to those from the control group. Defined in the standard space, both the forceps minor and corticospinal tract ROI templates were a close fit to the FA brain images from the INPH and control groups.

Figure 2 shows statistical comparisons of FA values in the INPH and control groups. Within both
ROIs, FA values were significantly lower in the INPH group than in the control group. Within the forceps minor, the mean FA value was 0.504 for the INPH group and 0.631 for the control group, indicating that mean FA for INPH patients was approximately $20 \%$ lower. In the corticospinal tracts, the mean FA value was 0.588 for the INPH group and 0.632 for the control group, showing an approximately $7 \%$ difference. FA values from the forceps minor provide stronger evidence of INPH than values from the corticospinal tracts.

Table 3 shows the correlations between FA values and clinical manifestations. FA values within the forceps minor were significantly correlated with urinary incontinence $(\mathrm{p}=0.023)$. Within this ROI, lower FA values tended to correlate with gait disturbance $(p=0.066)$. No such relationships were apparent after analyzing data from the corticospinal tracts.

\section{Discussion}

The present comparison of FA values in INPH patients and control subjects observed that INPH FA values within the forceps minor were approximately $20 \%$ lower. In addition, data analyses revealed a ten- 

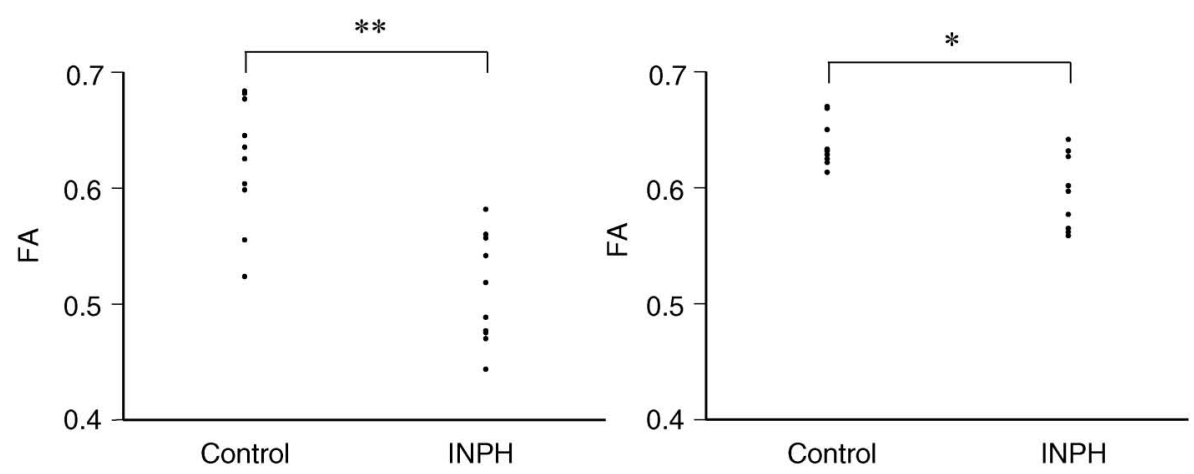

Fig. 2 Comparisons of fractional anisotropy (FA) values within the forceps minor (left) and corticospinal tracts (right) of patients with idiopathic normal pressure hydrocephalus $(\mathrm{INPH})$ and control subjects. ${ }^{*} p=0.0009,{ }^{*} p=$ 0.0001.

Table 3 Correlations between fractional anisotropy values and symptoms graded by idiopathic normal pressure hydrocephalus (INPH) grading scale and modified Rankin scale (mRS)

\begin{tabular}{llcc}
\hline Region of interest & \multicolumn{1}{c}{ Symptom grading } & Coefficient & $\mathrm{p}$ Value \\
\hline Forceps minor & mRS & -0.579 & 0.079 \\
& INPH grading scale & & \\
& cognitive impairment & -0.128 & 0.724 \\
& gait disturbance & -0.601 & 0.066 \\
Corticospinal tracts & urinary incontinence & $-\mathbf{0 . 7 0 5}$ & $\mathbf{0 . 0 2 3}$ \\
& mRS & -0.219 & 0.544 \\
& INPH grading scale & & \\
& cognitive impairment & 0.005 & 0.988 \\
& gait disturbance & -0.198 & 0.584 \\
& urinary incontinence & -0.269 & 0.452 \\
\hline
\end{tabular}

Bold indicates statistical significance.

dency for forceps minor FA values to decrease with more severe clinical manifestations of INPH.

The brain images of INPH patients usually show a wide range of individual variability because of ventricular dilation, and this has made inter-subject analysis of images technically difficult. ${ }^{35)}$ To meet this challenge, we attempted to apply computer-automated standardization of brain images. As shown in Fig. 1, this standardization could transform FA brain images from INPH patients into the standard space as successfully as images from the control group. We were then able to perform inter-subject analyses of FA brain images.

In our population, the mean age of the control group was slightly lower than that of the INPH group. Therefore, the observed differences in FA could be simply attributed to the age difference. Indeed, several reports have provided evidence that FA values naturally decrease with normal aging. ${ }^{10,26)}$ However, our findings show an approximately $20 \%$ decrease in FA values within the forceps minor of the INPH patients. A difference of this magnitude cannot be attributed to the influence of normal aging. ${ }^{10,26)}$

Several previous studies have shown that INPH patients exhibit lower FA values in the frontal areas, including the forceps minor, than normal subjects. ${ }^{6,27)}$ In addition, our analyses revealed some association of lower FA values in these areas with the clinical symptoms of INPH (Table 3). Taken together, these findings suggest that neural degeneration in these areas could contribute to the essential pathology that causes INPH symptoms. Therefore, FA values could be used for the diagnosis of INPH, but a careful study of more subjects is needed to define a cut-off point and assess the sensitivity and specificity.

Slightly, but statistically significantly, lower FA values were observed within the corticospinal tracts. This finding conflicts with previous studies that reported higher FA values within the corticospinal tracts for INPH patients than for normal subjects. ${ }^{6,22)}$ Such changes were even reversible after shunt implantation. ${ }^{22}$ This could mean that higher corticospinal tract FA values may be important to better characterize INPH white matter changes. Recently, DTI tractography showed that neural fibers in corticospinal tracts were compressed by ventricular dilation, resulting in higher FA values for INPH patients. ${ }^{7)}$ The discrepancy may be attributed to the differences in the processes for the brain image analyses, as our study employed spatial transformation into the standard brain. Further study is required to explain these discrepancies.

Regarding the effectiveness of the tap test, the Japanese Guidelines for INPH (2004) stipulate a more than $10 \%$ improvement in the up-and-go test. ${ }^{4)}$ In the present study, all tested patients except for 
Case 3 met the stipulation: Case 3 also achieved the best initial results (10 sec for 15 steps), and ceiling effects are likely to account for the lack of adequate improvement. ${ }^{30}$ Thus, we are confident that our population generally responded well to the tap test, confirming the reliability of our population sample.

This study has a number of limitations. First, the sample size was relatively small (10 persons each for the INPH and control groups). Even so, our analyses yielded statistically significant findings (Fig. 2 and Table 3). Second, we recruited patients with no history of Parkinson's disease, Alzheimer's disease, or other geriatric neural disorders. However, we often encounter patients with overlapping histories of multiple geriatric neural disorders in clinical practice. To better inform clinical practice, comparative studies including these neural disorders is needed. ${ }^{20)}$ Third, this study did not further analyze the relationships between FA values and the effects of the tap test. Although the Japanese Guidelines for INPH (2004) stipulate criteria, their validity and reliability have yet to be established.4) Future studies are needed to clarify this issue.

In summary, this study showed that FA values within the forceps minor were significantly lower in INPH patients and tended to correlate with the severity of clinical symptoms. This observation suggests that DTI may prove clinically useful in the diagnosis of INPH.

\section{Acknowledgment}

This research was supported by the Ministry of Health, Labour and Welfare of Japan (2008-NanchiGeneral-017).

\section{References}

1) Adams RD, Fisher CM, Hakim S, Ojemann RG, Sweet WH: Symptomatic occult hydrocephalus with "normal" cerebrospinal-fluid pressure. A treatable syndrome. N Engl J Med 273: 117-126, 1965

2) Dubois B, Slachevsky A, Litvan I, Pillon B: The FAB: a Frontal Assessment Battery at bedside. Neurology 55: 1621-1626, 2000

3) Folstein MF, Folstein SE, McHugh PR: "Mini-mental state'. A practical method for grading the cognitive state of patients for the clinician. J Psychiatr Res 12: 189-198, 1975

4) Guidelines Committee of Idiopathic Normal Pressure Hydrocephalus, the Japanese Society of Normal Pressure Hydrocephalus (ed): [Clinical Guidelines for Idiopathic Normal Pressure Hydrocephalus]. Osaka, Medical Review, 2004 (Japanese)

5) Hashimoto $M$, Ishikawa $M$, Mori E, Kuwana N; Study of INPH on neurological improvement
(SINPHONI): Diagnosis of idiopathic normal pressure hydrocephalus is supported by MRI-based scheme: a prospective cohort study. Cerebrospinal Fluid Res 7: 18, 2010

6) Hattingen E, Jurcoane A, Melber J, Blasel S, Zanella FE, Neumann-Haefelin T, Singer OC: Diffusion tensor imaging in patients with adult chronic idiopathic hydrocephalus. Neurosurgery 66: 917-924, 2010

7) Hattori T, Yuasa T, Aoki S, Sato R, Sawaura H, Mori $\mathrm{T}$, Mizusawa $\mathrm{H}$ : Altered microstructure in corticospinal tract in idiopathic normal pressure hydrocephalus: comparison with Alzheimer disease and Parkinson disease with dementia. AJNR Am J Neuroradiol 32: 1681-1687, 2011

8) Hebb AO, Cusimano MD: Idiopathic normal pressure hydrocephalus: a systematic review of diagnosis and outcome. Neurosurgery 49: 1166-1186, 2001

9) Hong YJ, Yoon B, Shim YS, Cho AH, Lim SC, Ahn KJ, Yang DW: Differences in microstructural alterations of the hippocampus in Alzheimer disease and idiopathic normal pressure hydrocephalus: a diffusion tensor imaging study. AJNR Am J Neuroradiol 31: 1867-1872, 2010

10) Hsu JL, Van Hecke W, Bai CH, Lee CH, Tsai YF, Chiu HC, Jaw FS, Hsu CY, Leu JG, Chen WH, Leemans A: Microstructural white matter changes in normal aging: a diffusion tensor imaging study with higher-order polynomial regression models. Neuroimage 49: 32-43, 2010

11) Image Analysis Group, Functional MRI of the Brain (FMRIB): FSLUTILS. Miscellaneous FSL Image Utilities. Oxford, UK, Oxford Center for FMRIB, (c) 2005-2012. Available from: http://fsl.fmrib.ox.ac. uk/fsl/avwutils/index.html. Accessed April 15, 2011

12) Image Analysis Group, Functional MRI of the Brain (FMRIB): FSLView v3.1. Oxford, UK, Oxford Center for FMRIB, (C) 2005-2012. Available from: http://www.fmrib.ox.ac.uk/fsl/fslview/index.html. Accessed April 15, 2011

13) Image Analysis Group, Functional MRI of the Brain (FMRIB): FNIRT-FMRIB's Non-linear Image Registration Tool. FNIRT v1.0\&beta-User Guide. Oxford, UK, Oxford Center for FMRIB, @ 2005-2012. Available from: http://www.fmrib.ox.ac.uk/fsl/fnirt/index. html. Accessed April 15, 2011

14) Image Analysis Group, Functional MRI of the Brain (FMRIB): FLIRT-FMRIB's Linear Image Registration Tool. FLIRT v5.5-User Guide. Oxford, UK, Oxford Center for FMRIB, (C) 2005-2012. Available from: http://www.fmrib.ox.ac.uk/fsl/flirt/. Accessed April 15, 2011

15) Image Analysis Group, Functional MRI of the Brain (FMRIB): BET-Brain Extraction Tool. BET v2.1User Guide. Oxford, UK, Oxford Center for FMRIB, (C) 2005-2012. Available from: http://www.fmrib.ox. ac.uk/fsl/bet2/index.html. Accessed April 15, 2011

16) Image Analysis Group, Functional MRI of the Brain (FMRIB): FMRIB Software Library. FSL 4.1 August 2008. Oxford, UK, Oxford Center for FMRIB, 
(c) 2005-2012. Available from: http://www.fmrib.ox. ac.uk/fsl/. Accessed April 15, 2011

17) Image Analysis Group, Functional MRI of the Brain (FMRIB): FMRIB's Diffusion Toolbox-FDT v2.0. Oxford, UK, Oxford Center for FMRIB, (C) 2005-2012. Available from: http://www.fmrib.ox.ac.uk/fsl/fdt/ index.html. Accessed April 15, 2011

18) Ishii $K$, Kawaguchi $T$, Shimada $K$, Ohkawa $S$, Miyamoto N, Kanda T, Uemura T, Yoshikawa T, Mori E: Voxel-based analysis of gray matter and CSF space in idiopathic normal pressure hydrocephalus. Dement Geriatr Cogn Disord 25: 329-335, 2008

19) Johns Hopkins Medical Institute Laboratory of Brain Anatomical MRI: Human White Matter Anatomy and Development. Baltimore, Johns Hopkins Medical Institute Laboratory of Brain Anatomical MRI. Available from: http://cmrm.med.jhmi.edu/. Accessed April 15, 2011

20) Kanno S, Abe N, Saito M, Takagi M, Nishio $Y$, Hayashi A, Uchiyama M, Hanaki R, Kikuchi H, Hiraoka K, Yamasaki H, Iizuka O, Takeda A, Itoyama Y, Takahashi S, Mori E: White matter involvement in idiopathic normal pressure hydrocephalus: a voxelbased diffusion tensor imaging study. J Neurol 258: 1949-1957, 2011

21) Kazui H, Mori E, Hashimoto M, Ishikawa M, Hirono N, Takeda M: Effect of shunt operation on idiopathic normal pressure hydrocephalus patients in reducing caregiver burden: evidence from SINPHONI. Dement Geriatr Cogn Disord 31: 363-370, 2011

22) Kim MJ, Seo SW, Lee KM, Kim ST, Lee JI, Nam DH, Na DL: Differential diagnosis of idiopathic normal pressure hydrocephalus from other dementias using diffusion tensor imaging. AJNR Am J Neuroradiol 32: 1496-1503, 2011

23) Koyama T, Tsuji M, Miyake H, Ohmura T, Domen K: Motor outcome for patients with acute intracerebral hemorrhage predicted using diffusion tensor imaging: an application of ordinal logistic modeling. J Stroke Cerebrovasc Dis Epub 2011 Apr 19

24) Krauss JK, Regel JP, Vach W, Orszagh M, Jungling FD, Bohus M, Droste DW: White matter lesions in patients with idiopathic normal pressure hydrocephalus and in an age-matched control group: a comparative study. Neurosurgery 40: 491-496, 1997

25) Kubo Y, Kazui H, Yoshida T, Kito Y, Kimura N, Tokunaga H, Ogino A, Miyake H, Ishikawa M, Takeda M: Validation of grading scale for evaluating symptoms of idiopathic normal-pressure hydrocephalus. Dement Geriatr Cogn Disord 25: 37-45, 2008

26) Lee CE, Danielian LE, Thomasson D, Baker EH: Normal regional fractional anisotropy and apparent diffusion coefficient of the brain measured on a $3 \mathrm{~T}$ MR scanner. Neuroradiology 51: 3-9, 2009

27) Lenfeldt N, Larsson A, Nyberg L, Birgander R, Ek- lund A, Malm J: Diffusion tensor imaging reveals supplementary lesions to frontal white matter in idiopathic normal pressure hydrocephalus. Neurosurgery 68: 1586-1593, 2011

28) Mori K: Management of idiopathic normal-pressure hydrocephalus: a multiinstitutional study conducted in Japan. J Neurosurg 95: 970-973, 2001

29) Mori S, Oishi K, Jiang H, Jiang L, Li X, Akhter K, Hua K, Faria AV, Mahmood A, Woods R, Toga AW, Pike GB, Neto PR, Evans A, Zhang J, Huang H, Miller MI, van Zijl P, Mazziotta J: Stereotaxic white matter atlas based on diffusion tensor imaging in an ICBM template. Neuroimage 40: 570-582, 2008

30) Podsiadlo D, Richardson S: The timed "Up \& Go": a test of basic functional mobility for frail elderly persons. J Am Geriatr Soc 39: 142-148, 1991

31) Relkin N, Marmarou A, Klinge P, Bergsneider M, Black PM: Diagnosing idiopathic normal-pressure hydrocephalus. Neurosurgery 57(3 Suppl): S4-16, ii-v, 2005

32) Tarnaris A, Kitchen ND, Watkins LD: Noninvasive biomarkers in normal pressure hydrocephalus: evidence for the role of neuroimaging. J Neurosurg 110: 837-851, 2009

33) van Swieten JC, Koudstaal PJ, Visser MC, Schouten $\mathrm{HJ}$, van Gijn J: Interobserver agreement for the assessment of handicap in stroke patients. Stroke 19: 604-607, 1988

34) Woolrich MW, Jbabdi S, Patenaude B, Chappell M, Makni S, Behrens T, Beckmann C, Jenkinson M, Smith SM: Bayesian analysis of neuroimaging data in FSL. Neuroimage 45(1 Suppl): S173-186, 2009

35) Yamashita F, Sasaki M, Takahashi S, Matsuda H, Kudo K, Narumi S, Terayama Y, Asada T: Detection of changes in cerebrospinal fluid space in idiopathic normal pressure hydrocephalus using voxel-based morphometry. Neuroradiology 52: 381-386, 2010

36) Yoshioka H, Horikoshi T, Aoki S, Hori M, Ishigame K, Uchida M, Sugita M, Araki T, Kinouchi H: Diffusion tensor tractography predicts motor functional outcome in patients with spontaneous intracerebral hemorrhage. Neurosurgery 62: 97-103, 2008

37) Yu C, Zhu C, Zhang Y, Chen H, Qin W, Wang M, Li $\mathrm{K}$ : A longitudinal diffusion tensor imaging study on Wallerian degeneration of corticospinal tract after motor pathway stroke. Neuroimage 47: 451-458, 2009

Address reprint requests to: Tetsuo Koyama MD, PhD, Department of Rehabilitation Medicine, Nishinomiya Kyoritsu Neurosurgical Hospital, 11-1 Imazu-Yamanaka-cho, Nishinomiya, Hyogo 663-8211, Japan.

e-mail: ytkoyama@bd6.so-net.ne.jp 